\title{
Option pricing in a Garch model with Tempered Stable innovations
}

\author{
Lorenzo Mercuri*
}

October, 1, 2007

\begin{abstract}
The key problem for option pricing in Garch models is that the risk neutral distribution of the underlying is known in explicit form only one day ahead and not at maturity. This problem was solved in the HestonNandi model (1997), where it is possible to compute the characteristic function of the underlying by a recursive procedure and options can be priced by Inverse Fourier Transform, see Heston (1993). Following the same idea, Christoffersen Heston and Jacobs (2004) proposed a Garchlike model with Inverse Gaussian Innovations and recently Bellini and Mercuri (2007) obtained a similar recursive procedure in a model with Gamma innovations.

In this work, we present a new Garch-like model with Tempered Stable innovations that encompasses both the CJH and the BM models as special cases. As it is costumary for this class of models, the pricing measure is chosen by means of the Conditional Esscher Transform (Siu et al. 2004). The TS model is calibrated on SP500 closing option prices and its performance is compared with the CJH, the BM and the Heston Nandi models.
\end{abstract}

Keywords Option Pricing, Garch model, Tempered Stable distribution, Semi-analytical valuation

\section{Introduction}

It is well known that, from an empirical point of view, the Black-Scholes model is not able to capture some "stylized facts" such as skewness, heavy tails and volatility clustering that are observed in real financial time series. To model these stylized phenomena, Mandelbrot (1963) proposed the use of $\alpha$-Stable distributions. Unfortunately this class of distributions has infinite variance and hence the tails might be too heavy to model real financial data.

In order to gain more adaptability, a new family of distributions, called Tempered Stable $(T S)$, was obtained by multiplying the density of the positively

*Dipartimento Metodi Quantitativi per le Scienze Economiche ed Aziendali, Università degli Studi di Milano-Bicocca. E-mail: lorenzo.mercuri@unimib.it 
skewed $\alpha$-Stable $(0<\alpha<1)$ with an exponential function. In this way, the tails are thinner than $\alpha$-Stable but heavier than normal. This class was first introduced by Tweedie (1984), see also Hougaard (1986) for applications in survival analysis. This kind of distributions was also used by Barndorff-Nielsen and Shephard (2002) to build an Ornstein-Uhlenbeck-based model.

Recently, Rosìnski (2006) generalized the Tempered Stable distributions and classified them according to their Levy measure. In this way the Tempered Stable family admits a parametrization similar to the Stable distributions one; with this generalization it is also possible to obtain distributions with the whole real axis as support.

Kim et al. (2006) applied the Tempered Stable defined on the real axis to the Garch option pricing model but unfortunately the price for a call option must be evaluated by using MonteCarlo simulations.

Garch-like models are useful way to capture the aforementioned "stylized facts", therefore it is natural to apply these models for option pricing issues. In order to compute the price of a European call option, we need to know the riskneutral distribution of the underlying at maturity, but usually we know it only one day ahead. This problem was solved by Heston and Nandi (2003). They proposed a Garch-like model with normal innovations where it is possible to compute the characteristic function of the underlying by a recursive procedure and price options by Inverse Fourier Transform (Heston (1993)).

Unfortunately the Heston-Nandi model (HN) is not sufficiently flexible to explain some observed option biases, in particular when we consider options with very short maturities. As suggested by Christoffersen et al. (2004), this is due to the fact that the single period innovations are normal in these model. For this reason, Christoffersen et al. proposed a model with Inverse Gaussian innovations (CJH model) and recently Bellini and Mercuri (2007) built a model with Gamma innovations. These new Garch-like processes are able to capture the conditional Skewness and the conditional Kurtosis; moreover it is possible to obtain a recursive procedure for the evaluation of the characteristic function.

In this work, we present a new Garch-like model with Tempered Stable (proposed by Tweedie (1984)) innovations that allows a semi-analytical formula for option pricing. Moreover our model incorporates, as special cases, the BM and CJH model.

The paper is organized in the following way: in the first section we review some classical results about the Tempered Stable, in the second we present the model, we show how to determine an equivalent martingale measure and to obtain a recursive procedure for the characteristic function. In the last section we present the empirical results of the calibration on real data.

\section{Tempered Stable distributions}

Before introducing our Garch-like model with Tempered Stable innovations, we review some classical results. It is well known that the $\alpha$-Stable distributions have infinite $p-t h$ order moments for all $p>\alpha$ because the tails are too heavy. 
In order to have all moments finite, it is possible to "temper" the tails with the exponential function. This new class of distributions, called Tempered Stable $T S$, was introduced by Tweedie.

Let $s(x ; \alpha, \beta, c, \delta)$ be the density function of an $\alpha$-Stable distribution, where $\alpha \epsilon(0,2]$ is the characteristic exponent, $\beta \epsilon[-1,1]$ is the skewness parameter, $c$ $\epsilon(0,+\infty)$ is the scale parameter and $\delta \epsilon(-\infty,+\infty)$ is the location parameter. We say that an $\alpha$-Stable distribution is positively skewed if $\beta=1$. Notice that if $\beta=1$ and $\delta=0$ then the density function is defined on $[0,+\infty)$. It's possible to show that the Laplace Transform of a positively skewed $\alpha$-Stable is given by (See Carr and Wu (2003) or Zolotarev (1986)):

$$
E[\exp (-\theta X)]=\exp \left[-\theta \delta-\theta^{\alpha} c^{\alpha} \sec \left(\alpha \frac{\pi}{2}\right)\right]
$$

where $\sec (\theta)$ is the secant function defined as $\sec (\theta)=\frac{1}{\cos (\theta)}$.

Let $s\left(x ; \alpha, 1, \frac{a}{2^{\alpha} \sec \left(\alpha \frac{\pi}{2}\right)}, 0\right)$ be the positively skewed $\alpha$-Stable density function with $\alpha \in(0,1)$. We say that $T S(\alpha, a, b)$ is a Tempered Stable distribution with $\alpha \epsilon(0,1), a>0$ and $b \geq 0$, if its density function is given by

$$
p(x ; \alpha, a, b)=\exp (a b) s\left(x ; \alpha, 1, \frac{a}{2^{\alpha} \sec \left(\alpha \frac{\pi}{2}\right)}, 0\right) \exp \left(-\frac{1}{2} b^{1 / \alpha} x\right)
$$

We note that the $b$ parameter controls the tail behavior, indeed if $b$ is equal to zero we have a positively skewed $\alpha$-Stable. When $b$ increases we obtain a distribution with thinner tails as shown in the following pictures.

\section{Insert figure 1 here.}

Moreover, using (1) and (2), we obtain the characteristic function of the Tempered Stable:

$$
\begin{aligned}
E[\exp (i \theta X)] & =\exp \left[a b-a\left(b^{1 / \alpha}-2 i \theta\right)^{\alpha}\right]= \\
& =\exp \left[a b\left[1-\left(1-2 i \theta b^{-1 / \alpha}\right)^{\alpha}\right]\right]
\end{aligned}
$$

Remark 1 It is not difficult to see that the $I G(a, b)$ and $\operatorname{Gamma}\left(a_{1}, b_{1}\right)$ distributions are special cases of (3) if $\alpha=1 / 2$ and $\alpha \rightarrow 0, a=\frac{a_{1}}{\alpha} b=\left(2 b_{1}\right)^{\alpha}$ respectively. The convergence is illustrated in the following picture:

Insert figure 2 here. 
The first four moments are given by:

$$
\begin{aligned}
E(X) & =2 a \alpha b^{(\alpha-1) / \alpha} \\
\operatorname{Var}(X) & =4 a \alpha(1-\alpha) b^{(\alpha-2) / \alpha} \\
\operatorname{Skew}(X) & =(\alpha-2)(a b \alpha(1-\alpha))^{-1 / 2} \\
\text { Kurtosis }(X) & =3+[4 \alpha-6-\alpha(1-\alpha)][a b \alpha(1-\alpha)]^{-1}
\end{aligned}
$$

\section{The Model}

\subsection{The Tempered Stable Garch process}

We consider a market with two assets: the riskless asset given by

$$
B_{t}=B_{t-1} e^{r_{t}}
$$

where $r_{t}$ is a predictable process, for simplicity we assume constant.

The stock price:

$$
S_{t}=S_{t-1} \exp \left(X_{t}\right)
$$

where $X_{t}$ is the log-return process with the following dynamics under the real measure:

$$
X_{t}=r+\lambda h_{t}-\frac{Z_{t}}{2 \sqrt{\alpha a(1-\alpha) b^{(\alpha-2) / \alpha}}}
$$

The conditional distribution ${ }^{1}$ of $Z_{t}$ is $T S\left(\alpha, a h_{t}, b\right)$ and the process $h_{t}$ has the dynamics specified below:

$$
h_{t}=\alpha_{0}+\alpha_{1} \frac{Z_{t-1}}{2 \sqrt{\alpha a(1-\alpha) b^{(\alpha-2) / \alpha}}}+\beta_{1} h_{t-1}
$$

From the properties of the Tempered Stable, the conditional mean and conditional variance can be derived as:

$$
\begin{gathered}
E_{t-1}\left(X_{t}\right)=r+\left(\lambda-\frac{\sqrt{\alpha a b}}{\sqrt{(1-\alpha)}}\right) h_{t} \\
\operatorname{Var}_{t-1}\left(X_{t}\right)=h_{t}
\end{gathered}
$$

Moreover the unconditional variance is given by

$$
E\left(h_{t}\right)=\frac{\alpha_{0}}{1-\left(\alpha_{1}+\beta_{1}\right)}
$$

\footnotetext{
${ }^{1}$ If we want a zero-mean stock return innovation we consider $Z_{t}^{\prime}=Z_{t}-\frac{\sqrt{a \alpha b}}{\sqrt{(1-\alpha)}} h_{t}$ instead of $Z_{t}$
} 
This model also captures the conditional skewness and the conditional kurtosis often observed in financial time series, since we have:

$$
\begin{aligned}
\text { Skew }_{t-1}\left(X_{t}\right) & =\frac{(\alpha-2)}{\sqrt{\alpha a h_{t} b(1-\alpha)}} \\
\text { Kurtosis }_{t-1}\left(X_{t}\right) & =3+\frac{[4 \alpha-6-\alpha(1-\alpha)]}{\left[a h_{t} b \alpha(1-\alpha)\right]}
\end{aligned}
$$

\subsection{Change of measure}

Our model represents a discrete time incomplete market with an infinity of states of nature. Therefore, in order to price a contingent claim, we have the classical problem of choosing an equivalent martingale measure.

Looking at the functional form of moment generating function of Tempered Stable, a natural choice is the Conditional Esscher Transform proposed by Buhlmann et al. (1996) as a generalization of Esscher Transform when only the conditional distribution is known (see also Siu et al. (2004) and Bellini and Mercuri (2007) for applications of this approach when the log-return is a Garch-like process).

We define the conditional moment generating function:

$$
M_{X_{k} \mid F_{k-1}}(\theta)=E\left[\exp \left(\theta X_{k}\right) \mid F_{k-1}\right]
$$

the change of measure is identified by solving the Conditional Esscher equation:

$$
\frac{M_{X_{k} \mid F_{k-1}}\left(\theta_{t}^{*}+1\right)}{M_{X_{k} \mid F_{k-1}}\left(\theta_{t}^{*}\right)}=e^{r_{t}}
$$

By the m.g.f of TS the (9) becomes:

$$
\exp \left(-\lambda h_{t}\right)=\frac{E_{t-1}\left[\exp \left[-\left(\theta_{t}^{*}+1\right) \frac{Z_{t}}{2 \sqrt{\alpha a(1-\alpha) b^{(\alpha-2) / \alpha}}}\right]\right]}{E_{t-1}\left[\exp \left[-\theta_{t}^{*} \frac{Z_{t}}{2 \sqrt{\alpha a(1-\alpha) b^{(\alpha-2) / \alpha}}}\right]\right]}
$$

By substituting:

$$
u=-\frac{1}{2 \sqrt{\alpha a(1-\alpha) b^{(\alpha-2) / \alpha}}}
$$

in (10) we can write:

$$
\exp \left(-\lambda h_{t}\right)=\exp \left[a\left(\left(b^{1 / \alpha}-2 \theta_{t}^{*} u\right)^{\alpha}-\left(b^{1 / \alpha}-2\left(1+\theta_{t}^{*}\right) u\right)^{\alpha}\right)\right]
$$

An analytical solution for this equation does not exist; therefore we need to solve it numerically. 
One of the advantages of the Esscher transform is that the conditional m.g.f of the log-returns under the martingale measure can be obtained by the following relation:

$$
M_{X_{k} \mid F_{k-1}}^{\prime}(c)=\frac{M_{X_{k} \mid F_{k-1}}\left(\theta_{t}^{*}+c\right)}{M_{X_{k} \mid F_{k-1}}\left(\theta_{t}^{*}\right)}
$$

In our case, the formula (13) becomes

$$
M_{X_{k} \mid F_{k-1}}^{\prime}(c)=\exp \left[c\left(r+\lambda h_{t}\right)+a\left(b^{1 / \alpha}-2 \theta_{t}^{*} u\right)^{\alpha}\left(1-\left(1-\frac{2 c u}{b^{1 / \alpha}-2 \theta_{t}^{*} u}\right)^{\alpha}\right)\right]
$$

We note that, under the new measure, the innovation is distributed again as TS but with a new parameter $b^{\prime}=\left(b^{1 / \alpha}-2 \theta_{t}^{*} u\right)^{\alpha}$ and the conditional variance is a linear function of the conditional variance under real measure as:

$$
h_{t}^{\prime}=\operatorname{Var}_{t-1}^{\prime}\left(X_{t}\right)=\left(\frac{b^{\prime}}{b}\right)^{(\alpha-2) / \alpha} h_{t}
$$

then, by using equation (15), and imposing

$$
\left\{\begin{array}{c}
\lambda^{\prime}=\lambda\left(\frac{b}{b^{\prime}}\right)^{(\alpha-2) / \alpha}, a^{\prime}=a\left(\frac{b}{b^{\prime}}\right)^{(\alpha-2) / \alpha} \\
\alpha_{0}^{\prime}=\alpha_{0}\left(\frac{b^{\prime}}{b}\right)^{(\alpha-2) / \alpha}, \alpha_{1}^{\prime}=\alpha_{1}\left(\frac{b^{\prime}}{b}\right)^{(\alpha-2) / \alpha}
\end{array}\right.
$$

the model can be rewritten as

$$
\left\{\begin{array}{c}
X_{t}=r+\lambda^{\prime} h_{t}^{\prime}-\frac{Z_{t}}{2 \sqrt{\alpha a^{\prime}(1-\alpha)\left(b^{\prime}\right)^{(\alpha-2) / \alpha}}} \\
h_{t}^{\prime}=\alpha_{0}^{\prime}+\alpha_{1}^{\prime} \frac{Z_{t-1}}{2 \sqrt{\alpha a^{\prime}(1-\alpha)\left(b^{\prime}\right)^{(\alpha-2) / \alpha}}}+\beta_{1} h_{t-1}
\end{array}\right.
$$

where $Z_{t} \sim T S\left(\alpha, a^{\prime} h_{t}^{\prime}, b^{\prime}\right)$.

If we compare the model under real measure with the same under martingale measure we see that the functional form is similar but with a different specification of the parameters.

\subsection{Option pricing}

In the previous section we have seen how to use the conditional Esscher Transform for selecting an equivalent martingale measure. Moreover we proved that the conditional distribution one period ahead is again a Tempered Stable. In order to price an option, we need the conditional distribution of the underlying asset at a generic maturity $T$. Following Heston-Nandi's approach, we show how a recursive procedure for conditional characteristic function of log-price can be obtained and then a call option can be priced by Inverse Fourier Transform. Moreover we will see that our model contains, as special cases, the BM model and the CJH model with slight modifications in the variance dynamics. 
We claim that the conditional m.g.f. of log-price has the following form:

$$
E_{t}\left[\exp \left[\phi \ln \left(S_{T}\right)\right]\right]=S_{t}^{\phi} \exp \left[A(t, T, \phi)+B(t, T, \phi) h_{t+1}\right]
$$

therefore we assume that this equation holds at time $t$ and by the iteration property of conditional expectations we determine the conditional m.g.f. at time $t$ in the following way:

$$
\begin{aligned}
E_{t}\left[\exp \left[\phi \ln \left(S_{T}\right)\right]\right]= & E_{t}\left[S_{t+1}^{\phi} \exp \left[A(t+1, T, \phi)+B(t+1, T, \phi) h_{t+2}\right]\right]= \\
=S_{t}^{\phi} E_{t}[ & \exp \left[\phi r+A(t+1, T, \phi)+\alpha_{0} B(t+1, T, \phi)+\right. \\
& +\phi \lambda h_{t+1}+\beta_{1} B(t+1, T, \phi) h_{t+1}+ \\
& \left.\left.+\frac{\left(\alpha_{1} B(t+1, T, \phi)-\phi\right)}{2 \sqrt{\alpha a(1-\alpha)(b)^{(\alpha-2) / \alpha}}} Z_{t+1}\right]\right]
\end{aligned}
$$

By the m.g.f. of $T S$ we have:

$$
\begin{gathered}
E_{t}\left[\exp \left[\phi \ln \left(S_{T}\right)\right]\right]=S_{t}^{\phi} \exp \left[\phi r+A(t+1, T, \phi)+\alpha_{0} B(t+1, T, \phi)+\right. \\
+\phi \lambda h_{t+1}+\beta_{1} B(t+1, T, \phi) h_{t+1}+ \\
\left.+a h_{t+1} b\left[1-\left(1-\frac{\left(\alpha_{1} B(t+1, T, \phi)-\phi\right)}{\sqrt{\alpha b a(1-\alpha)}}\right)^{\alpha}\right]\right]
\end{gathered}
$$

If we compare equation (20) with equation (18) we obtain the following system for the coefficients $A(t, T, \phi), B(t, T, \phi)$ :

$$
\left\{\begin{array}{c}
A(t, T, \phi)=\phi r+A(t+1, T, \phi)+\alpha_{0} B(t+1, T, \phi) \\
B(t, T, \phi)=\phi \lambda+\beta_{1} B(t+1, T, \phi)+a b\left[1-\left(1-\frac{\left(\alpha_{1} B(t+1, T, \phi)-\phi\right)}{\sqrt{\alpha b a(1-\alpha)}}\right)^{\alpha}\right]
\end{array}\right.
$$

with terminal conditions

$$
\left\{\begin{array}{l}
A(T, T, \phi)=0 \\
B(T, T, \phi)=0
\end{array}\right.
$$

We complete this section by proving that the recursive system in the BM model can be obtained, as a special case, by setting $b=2^{\alpha}, a=\frac{a_{1}}{\alpha}$ and computing the following limit in the coefficient $B(t, T, \phi)$

$$
\begin{gathered}
\lim _{\alpha \rightarrow 0^{+}} 2^{\alpha} \frac{a_{1}}{\alpha} h_{t+1}\left[1-\left(1-\frac{\left(\alpha_{1} B(t+1, T, \phi)-\phi\right)}{\sqrt{\alpha 2^{\alpha} \frac{a_{1}}{\alpha}(1-\alpha)}}\right)^{\alpha}\right]= \\
=-a_{1} h_{t+1} \ln \left(1+\frac{\phi-\alpha_{1} B(t+1, T, \phi)}{\sqrt{a_{1}}}\right)
\end{gathered}
$$


Moreover it is possible to obtain the recursive equations for coefficients in the CJH model by imposing $\alpha=\frac{1}{2}, a=\frac{1}{\eta^{2}}, b=1$ and considering the variance dynamics specified below:

$$
h_{t}=\alpha_{0}+\alpha_{1} Z_{t-1}+\beta_{1} h_{t-1}
$$

where $Z_{t}$ is conditionally distributed as $I G\left(\frac{h_{t}}{\eta^{2}}, 1\right)$.

\section{Calibrations and comparisons}

In this section we test the ability of our model to fit real option prices and compare it with the HN, CJH and the BM model. These models are defined by the following conditions:

- HN Model

$$
\left\{\begin{array}{l}
X_{t}=r-\frac{h_{t}}{2}+\varepsilon_{t}^{\prime} \\
\varepsilon_{t}^{\prime} \mid F_{t-1} \sim N\left(0, h_{t}\right) \\
h_{t}=\alpha_{0}+\frac{\alpha_{1}\left(\varepsilon_{t-1}^{\prime}-\left(\lambda+\frac{1}{2}+\gamma\right) h_{t-1}\right)^{2}}{h_{t-1}}+\beta_{1} h_{t-1}
\end{array}\right.
$$

- CJH Model

$$
\left\{\begin{array}{l}
X_{t}=r+\lambda h_{t}+\varepsilon_{t} \\
\varepsilon_{t} \mid F_{t-1}=\eta^{\prime} Y_{t}, \text { with } \eta^{\prime}=\frac{\eta}{\left[\frac{1}{\lambda \eta}+\frac{\eta^{2} \lambda}{2}\right]^{2}} \\
Y_{t} \sim I G\left(\delta_{t}^{\prime}\right) \text { with } \delta_{t}^{\prime}=\frac{h_{t}^{\prime}}{\eta^{\prime 2}} \\
h_{t}^{\prime}=\alpha_{0}^{\prime}+\beta_{1} h_{t-1}^{\prime}+\alpha_{1}^{\prime} \varepsilon_{t-1}+\frac{\gamma^{\prime} h_{t-1}^{\prime 2}}{\varepsilon_{t-1}}
\end{array}\right.
$$

- BM Model

$$
\left\{\begin{array}{l}
X_{t}=r+\lambda^{\prime} h_{t}^{\prime}+\varepsilon_{t} \\
\varepsilon_{t} \mid F_{t-1}=-\frac{1}{\sqrt{a_{1}^{\prime}}} Y_{t} \\
Y_{t} \sim G a\left(a_{1}^{\prime} h_{t}^{\prime}, 1\right) \text { with } \\
h_{t}^{\prime}=\alpha_{0}^{\prime}+\beta_{1} h_{t-1}^{\prime}+\alpha_{1}^{\prime} \varepsilon_{t}
\end{array}\right.
$$

We consider a dataset composed of 270 daily closing prices of European call options on S\&P500, and we identify each price by the triple $(t, K, T)$; where $t$ is the quotation day (varying from $06 / 22 / 06$ to $07 / 10 / 06$ ), $K$ is the strike price (9 equally spaced values ranging from 1250 to 1290) and $T$ is the maturity (July, August, September). The parameters of the models are determined by 
mean squared error minimization. That is, we minimize the difference between observed option price $C_{a l l}$ obs $(t, K, T)$ and the theoretical price $C_{a l l}$ theo $(t, K, T)$ :

$$
\underset{\theta}{\arg \min } M S Q(\theta)=\frac{1}{N} \sum_{t} \sum_{K} \sum_{T}\left[\operatorname{Call}_{\text {obs }}(t, K, T)-\text { Call }_{\text {theo }}(t, K, T)\right]^{2}
$$

where $\theta$ is the vector of parameters of the analyzed model. The minimization procedure is carried in MatLab environment.

The following table reports the calibrated parameters of the considered models:

\begin{tabular}{|l|l|l|l|l|}
\hline Parms & TS & CJH & BM & HN \\
\hline$\lambda^{\prime}$ & 147.29 & 110.6489 & 68.2202 & 21.685 \\
\hline$\alpha_{0}^{\prime}$ & $3.0217 \mathrm{e}-006$ & $1.7131 \mathrm{e}-005$ & $2.5773 \mathrm{e}-005$ & $4.7878 \mathrm{e}-006$ \\
\hline$\alpha_{1}^{\prime}$ & 0.00079467 & $5.1586 \mathrm{e}-005$ & 0.0067051 & $3.4539 \mathrm{e}-013$ \\
\hline$\beta_{1}$ & 0.82861 & 0.017339 & 0.00073407 & 0.88532 \\
\hline$\gamma^{\prime}$ & & 0.033306 & & \\
\hline$\eta^{\prime}$ & & 0.033306 & & \\
\hline$a_{1}^{\prime}$ & & & 4721.8 & \\
\hline$a^{\prime}$ & 12411 & & & \\
\hline$\alpha^{\prime}$ & 0.32834 & & & \\
\hline$b^{\prime}$ & 3.5999 & & & \\
\hline$R M S Q$ & 0.1212 & 0.1281 & 0.1315 & 0.1419 \\
\hline
\end{tabular}

As expected, our model achieves the smallest total root mean squared error $(R M S Q)$. In order to further investigate the pricing error, we consider the $R M S Q$ for each quotation day $t$ as:

$$
R M S Q(t, T)=\frac{1}{N_{K}} \sqrt{\sum_{K}\left[\operatorname{Call}_{\text {obs }}(t, K, T)-\operatorname{Call}_{\text {theo }}(t, K, T)\right]^{2}}
$$

and the $R M S Q$ for each strike price $K$ as:

$$
R M S Q(K, T)=\frac{1}{N_{t}} \sqrt{\sum_{K}\left[\operatorname{Call}_{\text {obs }}(t, K, T)-\operatorname{Call}_{\text {theo }}(t, K, T)\right]^{2}}
$$

where $N_{K}=9$ is the number of strike prices and $N_{t}=12$ is the number of days. Then in the following figure we plot the $R M S Q(t, T)$ and $R M S Q(K, T)$ for four models and for two maturities (July and August. We discarded September data since only a few quotations were available).

\section{Insert Figure 3 here}

The figure shows that our model outperforms quite uniformly the CJH and $\mathrm{BM}$ with respect to the strike and to the day. The situation is less clear if we 
compare it with the HN model. Indeed, for options with maturity July, the $R M S Q(K, T)$ of the HN model seems to be an increasing function of the strike price while in other cases the relation seems to be decreasing. For options with maturity August, we have the same upward and downward movements. In both cases, our model outperforms systematically the HN model only for options out of the money.

As observed in Bellini and Mercuri, we see also that the time dependence of average daily error is the same for all models considered. Moreover, our model seems to outperform the others if we consider options with very short maturities.

\section{Acknowledgements}

The author thanks Fabio Bellini, Carlo Sgarra, Chiara Pederzoli, Paolo Verzella and Giovanni Zambruno for their helpful comments. All remaining errors are responsibility of the author. 


\section{References}

[1] Bellini, F. Mercuri, L. (2007) "Option Pricing in Garch Models" Working paper n.124, Università degli studi di Milano - Bicocca, submitted.

[2] O.E. Barndorff-Nielsen, N. Shephard, (2002) "Normal modified stable processes" Theory Probab.Math. Statist. 65 1-20

[3] Buhlmann, H. Delbaen, F. Embrechts, P. Shiryaev, A.N. (1996) "No arbitrage, Change of Measure and Conditional Esscher Transform" CWI Quarterly 9(4) (1996) pp. 291-317

[4] Carr, Peter, and Liuren Wu (2003): "The Finite Moment Log Stable Process and Option Pricing," Journal of Finance, 58, 753-777.

[5] Christoffersen, P. Heston, S.L. Jacobs, C. (2006) "Option valuation with conditional skewness" Journal of Econometrics 131, 253-284

[6] Duan, J.C. (1995) "The Garch Option Pricing Model" Mathematical Finance 5, pp. 13-32

[7] Gerber, H.U. Shiu, E.S.W (1994) "Option pricing by Esscher transforms" Transactions of the Society of Actuaries 46 (1994) pp. 99-191

[8] Heston, S. (1993). "A Closed-Form Solution for Option With Stochastic Volatility with Applications to Bond and Currency Options" Review of Financial Studies 6, 327-343.

[9] Heston, S.L. Nandi, S. (2000) "A closed form option pricing model" Review of financial studies 13,3, pp. 585-562

[10] Hougaard, P. (1986) "Survival models for heterogeneous populations derived from stable distributions" Biometrika 73, pp.387-396.

[11] Kim S. Y., Rachev T.S., Bianchi M. L. and F.J. Fabozzi (2007) "A new Tempered Stable distribution and its application to finance" XL EWGFM

[12] Kim, Y. S., S. T. Rachev, and D. M. Chung, (2006). "The Modifed Tempered Stable Distribution, GARCH-Models and Option Pricing" Technical report, Chair of Econometrics, Statistics and Mathematical Finance School of Economics and Business Engineering University of Karlsruhe.

[13] Mandelbrot, B. B. (1963). "New methods in statistical economics" Journal of Political Economy, 71, 421-440.

[14] Rosinski, J. (2006). "Tempering Stable Processes" Working Paper, http://www.math.utk.edu/ rosinski/Manuscripts/tstableF.pdf.

[15] Shiryaev, A.N. (1999) "Essentials of stochastic finance: Facts, Models, Theory" World Scientific 
[16] Siu, T.K. Tong, H. Yang, H. (2004)"On pricing derivatives under Garch models: a dynamic Gerber-Shiu approach" North American Actuarial Journal 8(3) pp. 17-31

[17] Tweedie, M. (1984) " An index whichg distinguishes between some important exponantial families" In J. Ghosh and J. Roy (Eds.), Statistics: Applications and New Directions: Proc. Indian Statistical Institute Golden Jubilee International Conference, pp.579-604.

[18] Yang, H. (2004) "The Esscher transform" in Enciclopedia of actuarial sciences vol.2 pp. 617-621 New York Wile.

[19] Zolotarev, Vladimir M. (1986): "One-Dimensional Stable Laws" Vol. 65 in Translations of Mathematical Monographs. American Mathematical Society. (1983 Russian original). 

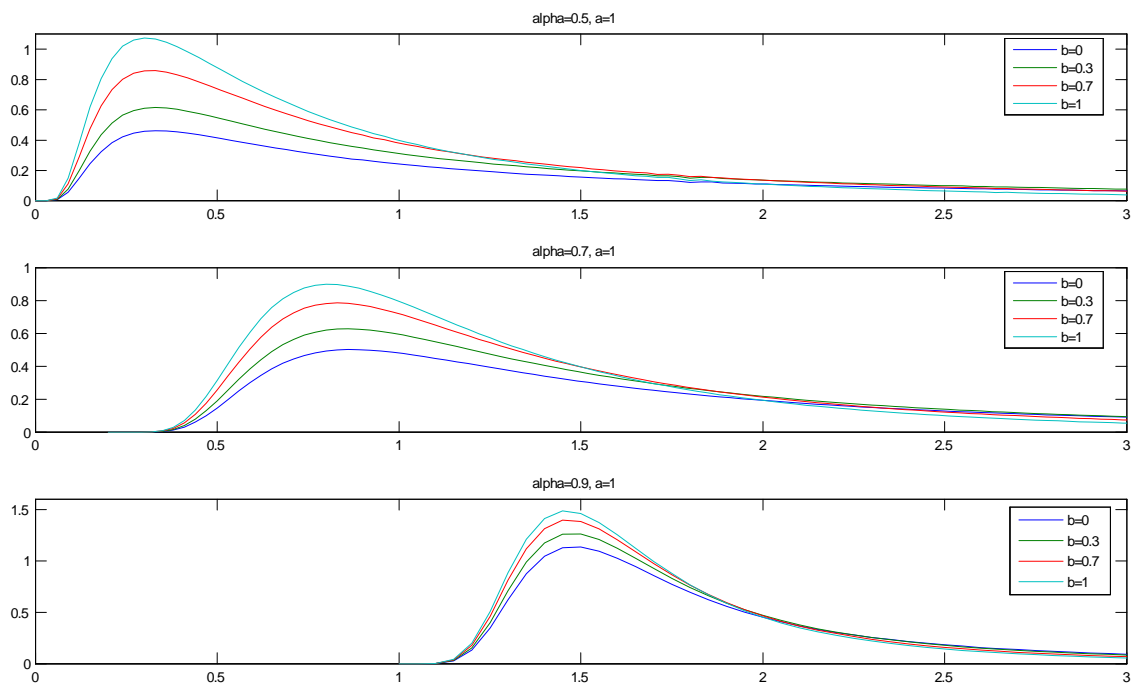

Figure 1: Behavior of the Tempered Stable density as function of $b$, for top to down $\alpha=0.5$, $\alpha=0.7$ and $\alpha=0.9, a=1$.
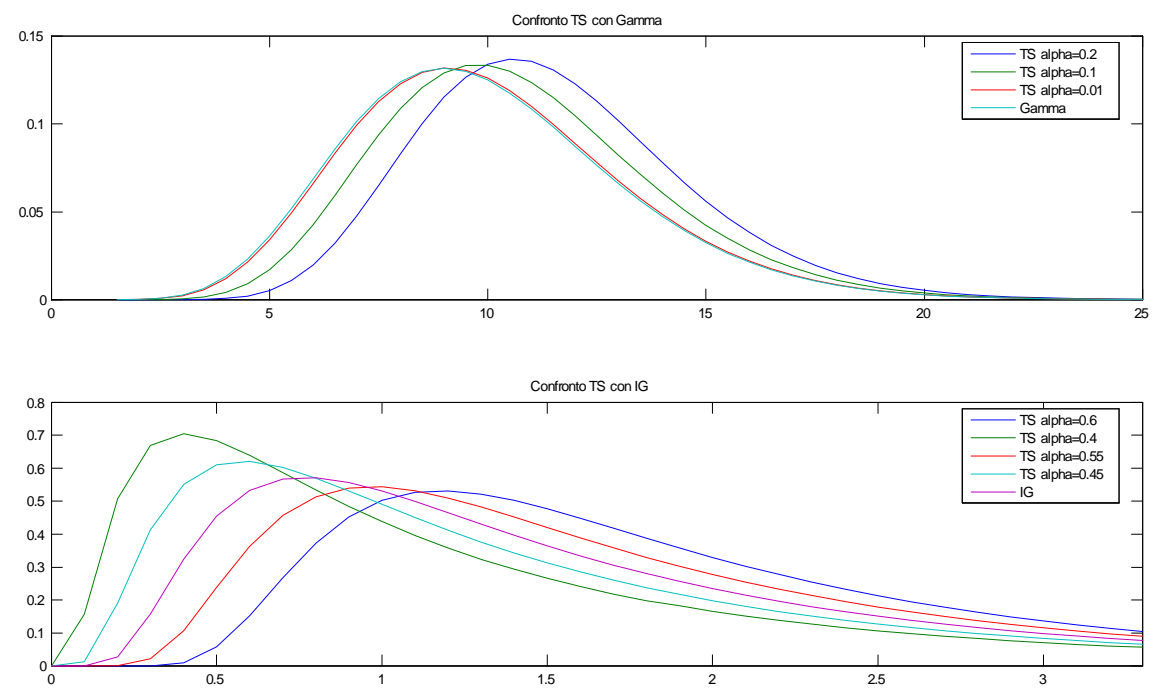

Figure 2: Convergence of the Tempered Stable density to the Gamma density (upper part) and the Inverse Gaussian (lower part). 

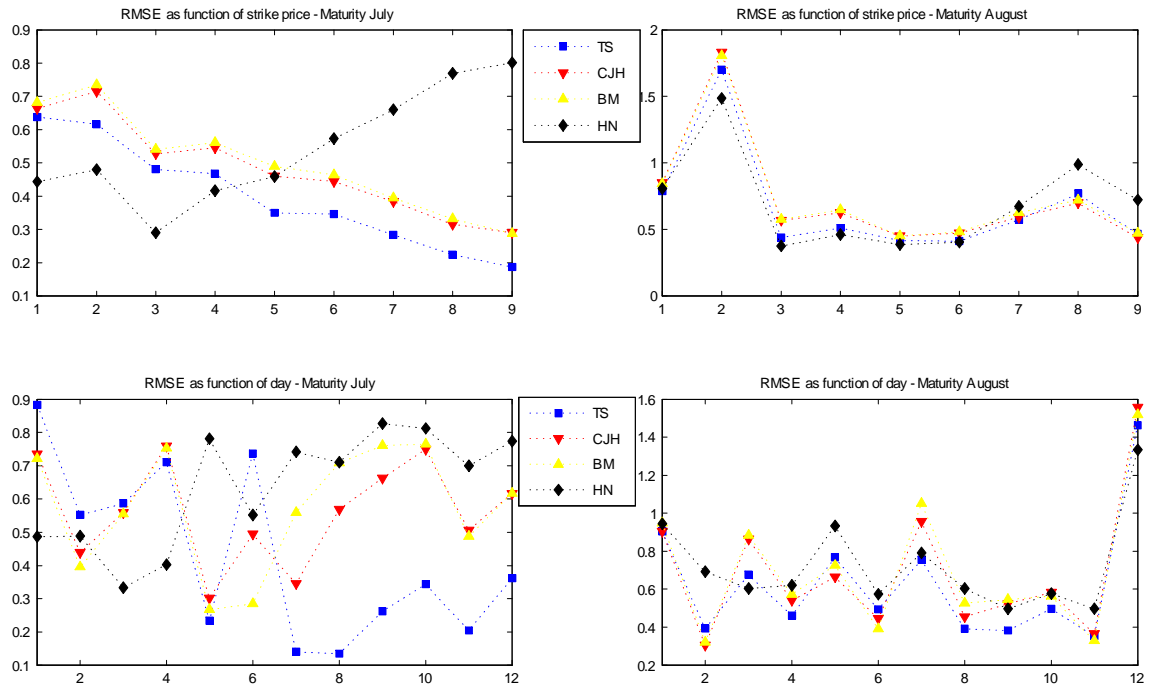

Figure 3: Dependence of the Root Mean Square Error (RMSE) on the strike price (upper part) and the day (lower part) for options with maturity July (right) and August (left). 